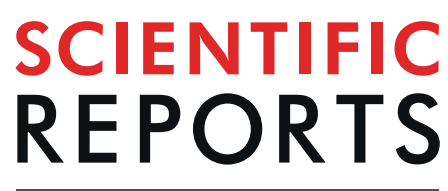

natureresearch

\title{
OPEN A comparative metabolomics analysis of the components of heartwood and sapwood in Taxus chinensis (Pilger) Rehd.
}

\author{
Fenjuan Shao ${ }^{1}$, Lisha Zhang ${ }^{1}$, Juan Guo ${ }^{2}$, Xiaochun Liu ${ }^{3}$, Wenhui Ma ${ }^{4}$, lain W. Wilson $\mathbb{1}^{5}$ \& \\ Deyou Qiu $\mathbb{1}^{1 *}$
}

Taxus chinensis is a well-known gymnosperm with great ornamental and medicinal value. Its purple red brown heartwood (HW) has many attributes such as straight texture, high density, mechanical strength, rich elasticity and corrosion resistance that is highly prized commercially. $T$. chinensis sapwood (SW), in comparison, lacks these important traits. At present, little is known about the differences of metabolites between the SW and HW in $T$. chinensis. Widely targeted metabolic profiling was performed to analyze the metabolic profiles of $\mathrm{HW}$ and $S W$ in $T$. chinensis using Liquid Chromatography-Electrospray lonization-Mass Spectrometry (LC-EI-MS). A total of 607 metabolites were detected in HW and SW. Among them, 146 metabolites were significantly higher, and 167 metabolites significantly lower, in $\mathrm{HW}$ as compared to SW. These differential metabolites were mainly involved in metabolic pathways and biosynthesis of secondary metabolites, such as flavonoids, flavone and flavonol, phenylpropanoids and antibiotics. Moreover, 71 flavonoids and isoflavones were found to be significantly different between $\mathrm{HW}$ and $\mathrm{SW}$. Our results show the difference of components between the $\mathrm{HW}$ and SW, which has potential significance to further elucidate the mechanism of $\mathrm{HW}$ color formation. The results will provide insight into the metabolites associated with wood color formation and useful information for understanding the metabolites associated with wood quality.

Wood, the secondary xylem of trees, is one of the most important source of materials and energy in the world ${ }^{1-3}$. It is important to human life, for example, wood can be as fuel for cooking as well as raw material for buildings ${ }^{2,3}$. In addition, it is also important to industrial, energy and environmental fields, such as renewable feedstock for pulp, biofuels, biomass energy and carbon $\operatorname{sink}^{2-4}$. Many tree species can be divided into sapwood (SW) and heartwood (HW) (Celedon and Bohlmann, 2018). HW is usually the dead inner wood, and reserve materials are converted into HW substances, while SW is the living, outermost portion wood that contains reserve materials ${ }^{5}$. Chemical composition plays an important role in wood properties, affecting physical and mechanical properties, natural durability, color and utilization of $\operatorname{wood}^{6,7}$. Compared to the SW, HW has an important economic value due to the natural decay resistance, wood color, wood fragrance and pharmaceutical substances ${ }^{8}$.

Wood color is an important trait for wood quality in the forest industry. It plays an important role in the processing of wood products, such as furniture, wood carving and building industries etc. It has been shown that the formation of wood color is due to the existence of secondary metabolites in the HW, including stilbenes, flavonoids and other phenolic compounds ${ }^{5}$, but the components of these secondary metabolites have not been investigated, and how these secondary metabolites affect wood color formation remain largely unknown. Predominately, research in wood color has mainly been focused on functional improvement through chemical methods ${ }^{9-11}$. The molecular genetic mechanisms of wood color formation are poorly understood. If the mechanism of wood color formation is understood, it may be possible to control wood color in trees via molecular genetics.

\footnotetext{
${ }^{1}$ State Key Laboratory of Tree Genetics and Breeding, Key Laboratory of Tree Breeding and Cultivation of National Forestry and Grassland Administration, Research Institute of Forestry, Chinese Academy of Forestry, Beijing, 100091, China. ${ }^{2}$ Research Institute of Wood Industry, Chinese Academy of Forestry, Beijing, 100091, China. ${ }^{3}$ Gansu Forestry and Grassland Administration, Lanzhou, 730030, China. 'Liangdang Forestry Administration, Gansu Province, 742400, China. ${ }^{5}$ CSIRO Agriculture and Food, PO Box 1700, Canberra, ACT, 2601, Australia. *email: qiudy@caf.ac.cn
} 


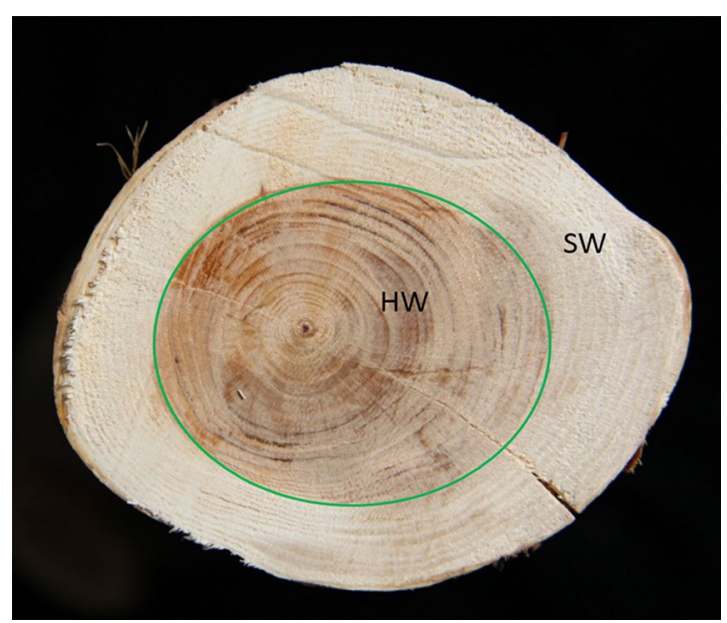

Figure 1. The heartwood and sapwood in T. chinensis (Pilger) Rehd. (HW: heartwood, SW: sapwood).

Taxus chinensis, belongs to the Taxus family, also known as yew, and is a well-known gymnosperm with great ornamental and medicinal value ${ }^{12}$. The bark of T. chinensis can produce paclitaxel, which has been widely used in the treatment of lung, ovarian and breast cancer ${ }^{13-15}$. In addition, the wood of Taxus has high commercial value because of its good aesthetic appearance, straight texture, high density, mechanical strength, rich elasticity, corrosion resistance, white yellowish SW and purple red brown colored HW. However, little is known about the metabolites variation between the SW and HW in T. chinensis.

Metabolomics is a valuable approach for the high-throughput and comprehensive study of complex metabolic compositions, and has been widely applied in plants ${ }^{16-20}$. Mass spectrometry methods have been used for detection and quantification of metabolites ${ }^{16-18}$. With the aim to investigate the metabolites variation between the SW and HW in T. chinensis, widely targeted metabolomics approach using liquid chromatography tandem mass spectrometry (LC-MS/MS) was performed. The metabolites in SW and HW were identified, the differences in metabolite profile in SW and HW compared. Our results are potentially useful for the further elucidation of the mechanism of HW color formation. The results will provide insight into the molecular mechanisms of wood formation and useful information for improved wood quality.

\section{Results and Discussion}

Metabolic profiling of heartwood and sapwood based on LC-MS/MS. SW and HW were collected from the branch from an approximately 30 -year-old of T. chinensis (Pilger) Rehd. The outer wood tissue with a pale yellow color is defined as SW and the central tissue with a red or dark-brown color is characterized as HW (Fig. 1). In order to investigate the components of HW and SW in T. chinensis, widely targeted metabolic profiling was performed to analyze the metabolic profiles of HW and SW in T. chinensis by using the Liquid Chromatography-Electrospray Ionization-Mass Spectrometry. Metabolomics data of HW and SW were processed using System Software Analyst (Version 1.6.3). Metabolites were quantitatively analyzed following collection of secondary data using the MRM model, as a result, a total of 607 metabolites were identified in HW and SW, including 52 lipids, 98 organic acids and their derivatives, 43 nucleotides and their derivatives, 132 flavonoids, 55 amino acids and their derivatives, 36 alkaloids, 93 phenylpropanoids, 12 vitamins, 17 terpenes, 21 carbohydrates and 48 others (supplementary file 1). These metabolites are involved in the most of primary and secondary metabolisms. Among these metabolites, the most abundant metabolites are the flavonoids, suggesting the flavonoids play a role in the process of the wood color formation.

Principal component analysis (PCA) and cluster analysis of HW and SW. In order to investigate the HW and SW metabolic differences and multivariate, mixed samples of the HW and SW were prepared and performed to metabolic profiling using the Liquid Chromatography-Electrospray Ionization-Mass Spectrometry. Principal component analysis (PCA) is used to reveal the differences in metabolic profiles between the HW and SW. In the PCA plot (Fig. 2A), the mixed samples grouped together, indicating that the mixed samples had similar metabolic profiles. PCA clearly grouped these samples into distinct clusters, indicating significant differences in metabolites between the HW and SW. Hierarchical cluster analysis (HCA) of the metabolites of the HW and SW was performed. The results of all detected metabolites are shown in a heatmap (Fig. 2B), which indicates the significant differences in the relative abundance of metabolites between HW and SW. The metabolite profile of the HW and SW are clearly divided into two main clusters based on the differences in accumulation patterns on the heatmap, suggesting a clear variation in terms of the metabolites abundance in the HW and SW.

Differential metabolite analysis based on partial least squares-discriminant analysis (PLS-DA). To obtain the metabolic differences between the HW and SW, the PLS-DA models was used to screen differential compounds between two groups of samples (Fig. 3). Moreover, a fold-change score $\geq 2$ or $\leq 0.5$ among the metabolites with a VIP value $>1$ was used to identify differential metabolites. The screening results have been illustrated using Volcano plots (Fig. 3). A total of 313 significant differences of metabolites were 

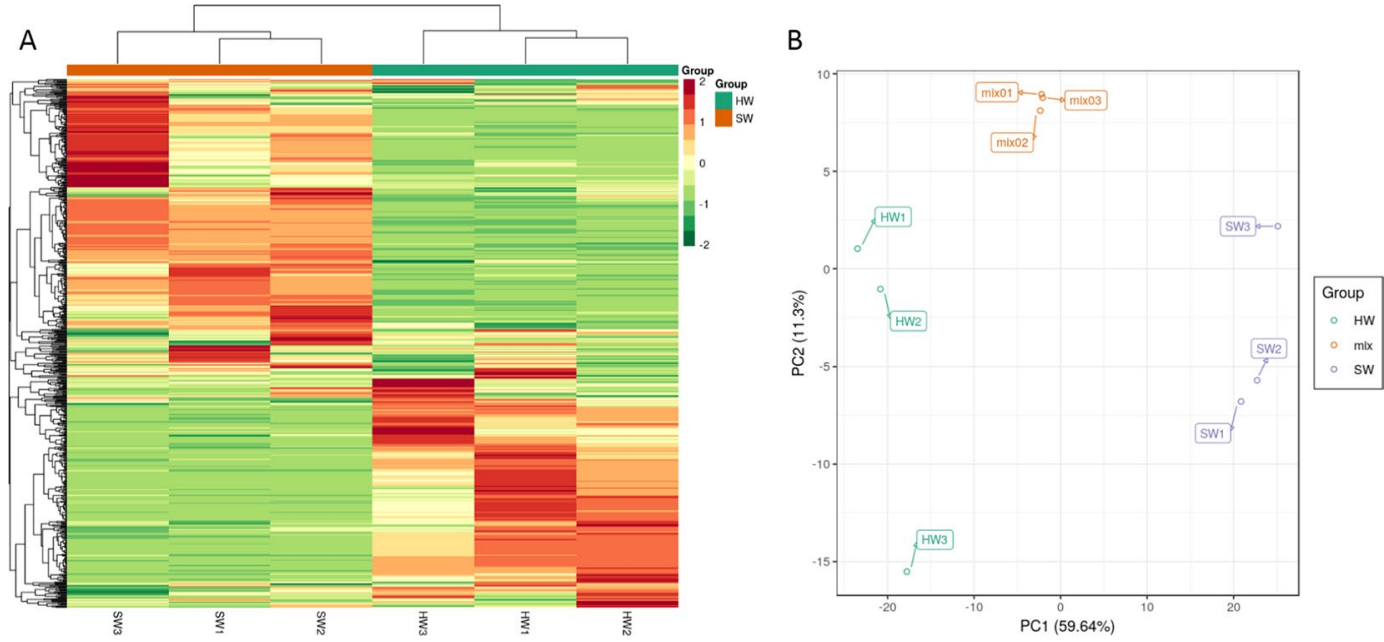

Figure 2. (A) Heatmap of the metabolites in heartwood and sapwood; (B) PCA score plot of the metabolites in the heartwood and sapwood.

A

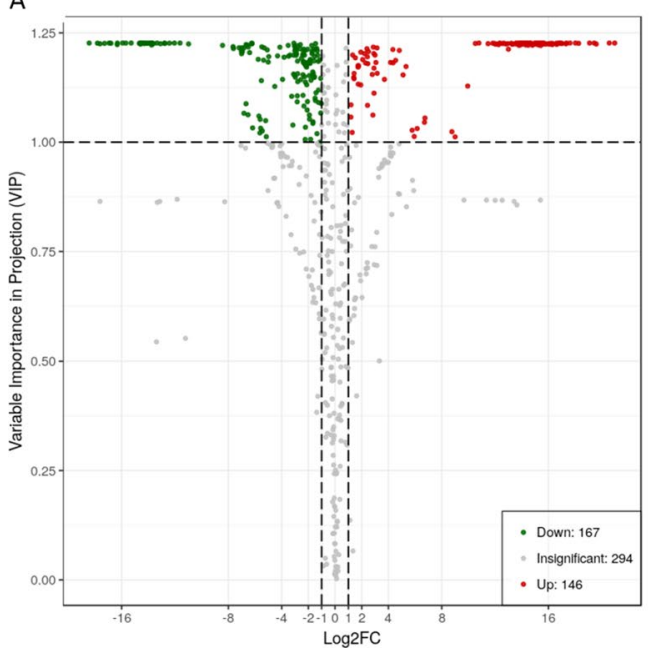

B

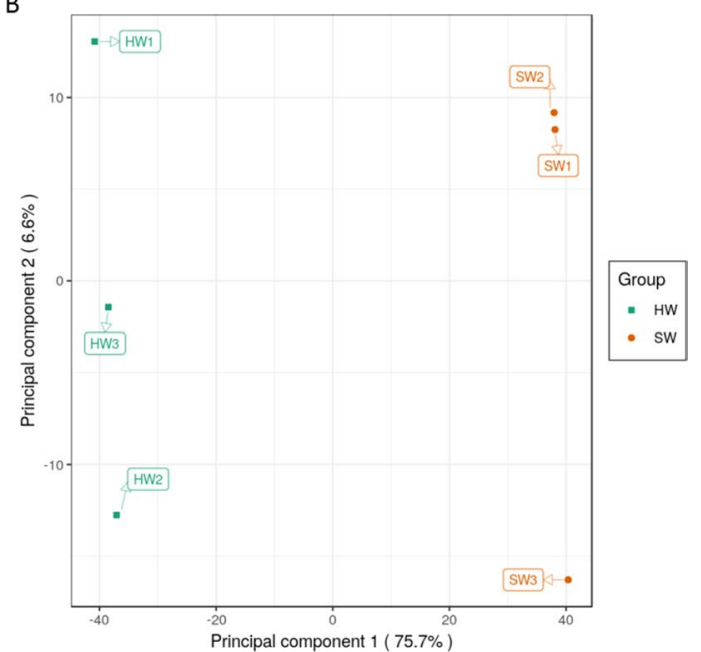

Figure 3. (A) The volcano plot of the differential metabolites in the heartwood and sapwood. Green dots represent down-regulated metabolites, red spots represent up-regulated metabolites and gray represent insignificant difference metabolites. (B) PLS-DA model plot of the differential metabolites in the heartwood and sapwood.

identified, of these differential metabolites there were 146 metabolites significantly higher and 167 metabolites significantly lower between the HW and SW (supplementary file 2). The top 30 metabolites that were higher in HW are shown in Table 1, and among them, 11 flavonoids were found to be significantly different between the HW and SW. These metabolites may be considered to be the representative differential metabolites for the HW and SW, influencing their wood properties especially the wood color.

Metabolic pathway analysis of differential metabolites. To obtain the pathway information of differential metabolites, the differential metabolites between the HW and SW were mapped to the Kyoto Encyclopedia of Genes and Genomes (KEGG) database (http://www.genome.jp/kegg/). The results are shown in Fig. 4, these differential metabolites are mainly involved in metabolic pathways and biosynthesis of secondary metabolites, such as flavonoids, flavone and flavonol, phenylpropanoids and antibiotics etc. Flavonoids, flavone and flavonol were included in phenylpropanoids, which are a large class of plant secondary metabolites derived from phenylalanine in plants. In addition to flavonoids, it also includes monolignols, phenolic acids, stilbenes, and coumarins $^{21-23}$. As we know, cellulose, hemicellulose and lignin are the major components of wood, but they do not exhibit color. It has been shown that the wood color is due to the existence of colored extractives contained in the wood. These colored extractives turn into dark color from a pale color by oxidation, polymerization and polymerization with wood main components over time ${ }^{24}$. Previous studies have shown that the extractives of 


\begin{tabular}{|c|c|c|c|c|c|}
\hline Number & Compounds & Class & VIP & Fold_Change & $\log 2 \mathrm{FC}$ \\
\hline 1 & C-pentosyl-apigenin O-p-coumaroylhexoside & Flavone & 1.227 & 2051851.852 & 20.968 \\
\hline 2 & $\beta$-Caryophyllene & Terpene & 1.227 & 1548148.148 & 20.562 \\
\hline 3 & Dehydrocorydaline & Alkaloids & 1.224 & 786666.667 & 19.585 \\
\hline 4 & Ayanin & Flavonol & 1.227 & 708148.148 & 19.434 \\
\hline 5 & Xanthotoxol & Phenylpropanoids & 1.227 & 555925.926 & 19.085 \\
\hline 6 & Vitamin A & Vitamins and derivatives & 1.226 & 314444.444 & 18.262 \\
\hline 7 & Ethyl 3,4-Dihydroxybenzoate & $\begin{array}{l}\text { Organic acids and } \\
\text { derivatives }\end{array}$ & 1.227 & 266296.296 & 18.023 \\
\hline 8 & Schizandrin B & Phenylpropanoids & 1.227 & 200740.741 & 17.615 \\
\hline 9 & N-Methyltryptamine & Alkaloids & 1.227 & 165925.926 & 17.340 \\
\hline 10 & Ellagic acid & Polyphenol & 1.225 & 162481.481 & 17.310 \\
\hline 11 & O-methylChrysoeriol 8-C-hexoside & Flavone & 1.226 & 131111.111 & 17.000 \\
\hline 12 & Malvidin 3-O-glucoside (Oenin) & Anthocyanins & 1.226 & 130888.889 & 16.998 \\
\hline 13 & Crocetin & Terpene & 1.226 & 119666.667 & 16.869 \\
\hline 14 & Chrysin O-hexoside & Flavone & 1.226 & 119259.259 & 16.864 \\
\hline 15 & 16-Hydroxy hexadecanoic acid & Lipids & 1.227 & 115148.148 & 16.813 \\
\hline 16 & Malvidin 3-O-galactoside & Anthocyanins & 1.225 & 101592.593 & 16.632 \\
\hline 17 & 4-Hydroxy-3,5-diisopropylbenzaldehyde & $\begin{array}{l}\text { Organic acids and } \\
\text { derivatives }\end{array}$ & 1.226 & 100814.815 & 16.621 \\
\hline 18 & Prehelminthosporolactone & Others & 1.224 & 100555.556 & 16.618 \\
\hline 19 & Ethyl cinnamate & Phenylpropanoids & 1.226 & 81074.074 & 16.307 \\
\hline 20 & Luteolin O-hexosyl-O-pentoside & Flavone & 1.223 & 80074.074 & 16.289 \\
\hline 21 & O-methylChrysoeriol 7-O-hexoside & Flavone & 1.226 & 75259.259 & 16.200 \\
\hline 22 & O-methylChrysoeriol 5-O-hexoside & Flavone & 1.224 & 69925.926 & 16.094 \\
\hline 23 & LysoPC 16:2 (2n isomer) & Lipids & 1.225 & 68037.037 & 16.054 \\
\hline 24 & 1,10-decanediol & Alcohols & 1.224 & 59000.000 & 15.848 \\
\hline 25 & 8-Methoxypsoralen & Phenylpropanoids & 1.226 & 54333.333 & 15.730 \\
\hline 26 & Psoralen & Phenylpropanoids & 1.224 & 51148.148 & 15.642 \\
\hline 27 & Myricetin & Flavonol & 1.226 & 50074.074 & 15.612 \\
\hline 28 & Glabridin & Flavonoid & 1.227 & 48370.370 & 15.562 \\
\hline 29 & Camptothecin & Alkaloids & 1.227 & 47222.222 & 15.527 \\
\hline 30 & Phytocassane C & Terpene & 1.226 & 46925.926 & 15.518 \\
\hline
\end{tabular}

Table 1. The top 30 significant differences of metabolites in heartwood and sapwood.
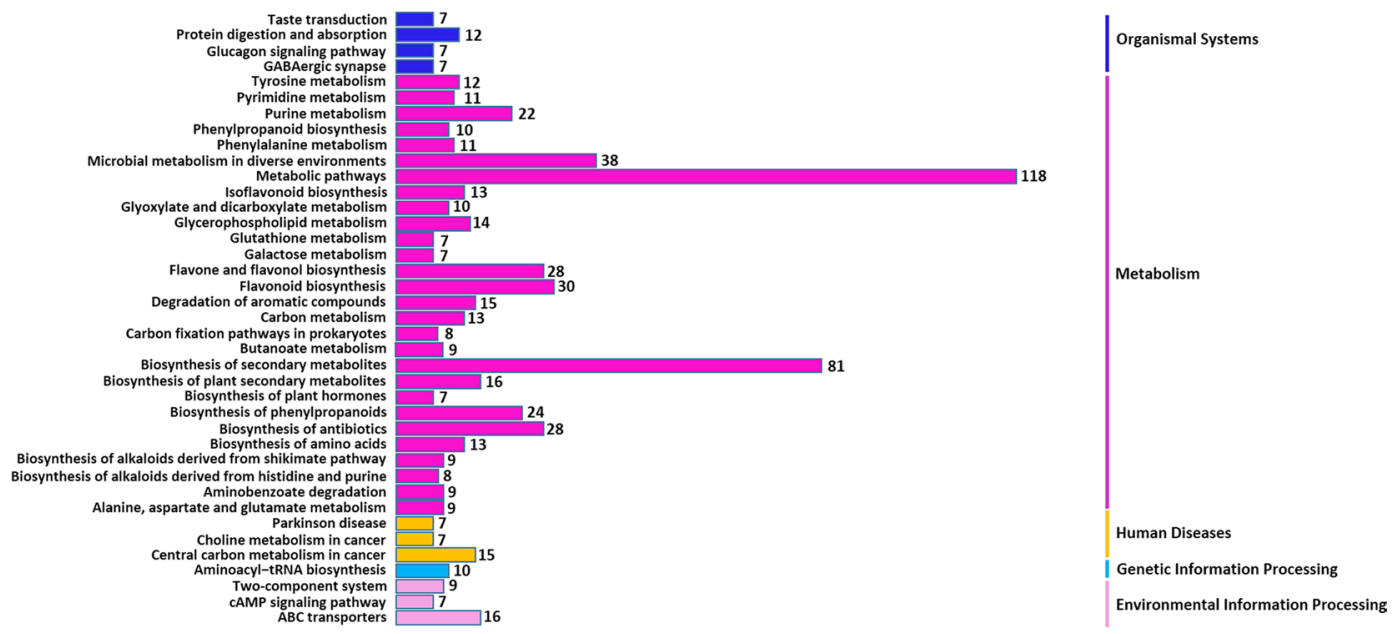

Figure 4. The distribution of metabolic pathways of the differential metabolites.

some important colored woods were the flavonoids ${ }^{25,26}$, suggesting that the flavonoid metabolites identified in this study may explain the difference in wood color between the HW and SW in T. chinensis.

Flavonoids identified in HW. Wood color is one of the important properties related to the wood utilization $^{24-26}$. Flavonoids as the major pigment molecules are widely studied in plants and it has been shown that 


\begin{tabular}{|c|c|c|c|c|c|}
\hline Compounds & Class & VIP & Fold_Change & $\log 2 \mathrm{FC}$ & Regulate \\
\hline Cyanidin 3-O-malonylhexoside & Anthocyanins & 1.227 & 0.00 & -14.63 & down \\
\hline O-methylChrysoeriol 5-O-hexoside & Flavone & 1.224 & 69925.93 & 16.09 & up \\
\hline Chrysoeriol 5-O-hexoside & Flavone & 1.226 & 0.00 & -14.29 & down \\
\hline Chrysin O-hexoside & Flavone & 1.226 & 119259.26 & 16.86 & up \\
\hline O-methylnaringenin C-pentoside & Flavone & 1.207 & 5.66 & 2.50 & up \\
\hline O-methylChrysoeriol 8-C-hexoside & Flavone & 1.226 & 131111.11 & 17.00 & up \\
\hline Ayanin & Flavonol & 1.227 & 708148.15 & 19.43 & up \\
\hline O-methylChrysoeriol 7-O-hexoside & Flavone & 1.226 & 75259.26 & 16.20 & up \\
\hline Isorhamnetin O-hexoside & Flavonol & 1.226 & 0.00 & -14.31 & down \\
\hline Selgin O-hexosyl-O-hexoside & Flavone & 1.226 & 25481.48 & 14.64 & up \\
\hline Luteolin O-hexosyl-O-pentoside & Flavone & 1.223 & 80074.07 & 16.29 & up \\
\hline Apigenin O-hexosyl-O-pentoside & Flavone & 1.223 & 4907.41 & 12.26 & up \\
\hline Chrysin 5-O-glucoside (Toringin) & Flavone & 1.031 & 71.33 & 6.16 & up \\
\hline Luteolin 3',7-di-O-glucoside & Flavone & 1.226 & 0.00 & -12.42 & down \\
\hline Isorhamnetin 5-O-hexoside & Flavonol & 1.227 & 0.00 & -14.51 & down \\
\hline Chrysoeriol 7-O-hexoside & Flavone & 1.227 & 0.00 & -14.34 & down \\
\hline C-hexosyl-isorhamnetin O-hexoside & Flavone & 1.227 & 32814.81 & 15.00 & up \\
\hline 6-C-hexosyl chrysoeriol O-hexoside & Flavone & 1.227 & 38962.96 & 15.25 & up \\
\hline C-pentosyl apigenin O-salicyloyl hexoside & Flavone & 1.223 & 38629.63 & 15.24 & up \\
\hline C-pentosyl-apigenin O-p-coumaroylhexoside & Flavone & 1.227 & 2051851.85 & 20.97 & up \\
\hline 8-C-hexosyl chrysoeriol O-hexoside & Flavone & 1.226 & 0.00 & -15.69 & down \\
\hline Tricin 5-O- $\beta$-guaiacylglycerol & Flavone & 1.173 & 39.99 & 5.32 & up \\
\hline Tricin O-rhamnoside & Flavone & 1.225 & 12848.15 & 13.65 & up \\
\hline Apigenin C-hexosyl-O-rutinoside & Flavone & 1.057 & 2.27 & 1.19 & up \\
\hline Tricin 7-O-hexoside & Flavone & 1.225 & 36296.30 & 15.15 & up \\
\hline Tricin 4'-O- $\beta$-guaiacylglycerol & Flavone & 1.212 & 8200.00 & 13.00 & up \\
\hline Tricin O-eudesmic acid & Flavone & 1.225 & 21555.56 & 14.40 & up \\
\hline Di-O-methylquercetin & Flavonol & 1.157 & 0.32 & -1.63 & down \\
\hline Kaempferol 7-O-rhamnoside & Flavonol & 1.210 & 0.21 & -2.25 & down \\
\hline Chrysoeriol & Flavone & 1.095 & 0.36 & -1.47 & down \\
\hline Kaempferol 3-O-rutinoside (Nicotiflorin) & Flavonol & 1.154 & 0.19 & -2.40 & down \\
\hline Naringenin & Flavanone & 1.182 & 17.86 & 4.16 & up \\
\hline Apigenin & Flavone & 1.202 & 0.30 & -1.72 & down \\
\hline Malvidin 3-O-galactoside & Anthocyanins & 1.225 & 101592.59 & 16.63 & up \\
\hline Malvidin 3-O-glucoside (Oenin) & Anthocyanins & 1.226 & 130888.89 & 17.00 & up \\
\hline Xanthohumol & Flavanone & 1.225 & 7825.93 & 12.93 & up \\
\hline Myricetin & Flavonol & 1.226 & 50074.07 & 15.61 & up \\
\hline Isorhamnetin 3-O-neohesperidoside & Flavonol & 1.156 & 0.14 & -2.80 & down \\
\hline Isorhamnetin & Flavonol & 1.144 & 13.00 & 3.70 & up \\
\hline 7-O-Methyleriodictyol & Flavanone & 1.073 & 0.23 & -2.09 & down \\
\hline Kaempferol 3-O-robinobioside (Biorobin) & Flavonol & 1.182 & 0.19 & -2.38 & down \\
\hline Isohemiphloin & Flavone & 1.226 & 3637.04 & 11.83 & up \\
\hline Hesperetin 7-rutinoside (Hesperidin) & Flavanone & 1.093 & 0.24 & -2.07 & down \\
\hline Naringenin chalcone & Flavanone & 1.181 & 20.49 & 4.36 & up \\
\hline Aromadedrin (Dihydrokaempferol) & Flavonol & 1.225 & 35185.19 & 15.10 & up \\
\hline 3-Hydroxyflavone & Flavonol & 1.225 & 36000.00 & 15.14 & up \\
\hline Liquiritigenin & Flavanone & 1.225 & 0.00 & -10.98 & down \\
\hline Biochanin A & Isoflavone & 1.062 & 0.26 & -1.92 & down \\
\hline 2'-Hydroxydaidzein & Isoflavone & 1.227 & 0.00 & -15.93 & down \\
\hline 2'-Hydroxygenistein & Isoflavone & 1.226 & 45962.96 & 15.49 & up \\
\hline Afzelechin (3,5,7,4'-Tetrahydroxyflavan) & Flavanone & 1.013 & 0.03 & -5.15 & down \\
\hline 3,7-Di-O-methylquercetin & Flavonol & 1.062 & 7.16 & 2.84 & up \\
\hline Prunetin & Isoflavone & 1.043 & 0.32 & -1.66 & down \\
\hline Tricetin & Flavone & 1.150 & 0.20 & -2.29 & down \\
\hline Rhamnetin (7-O-methxyl quercetin) & Flavonol & 1.227 & 16259.26 & 13.99 & up \\
\hline Fustin & Flavonol & 1.200 & 0.19 & -2.43 & down \\
\hline Rotenone & Isoflavone & 1.226 & 35777.78 & 15.13 & up \\
\hline
\end{tabular}




\begin{tabular}{|l|l|l|r|r|l|}
\hline Compounds & Class & VIP & Fold_Change & Log2FC & Regulate \\
\hline Homoeriodictyol & Flavanone & 1.150 & 0.34 & -1.55 & down \\
\hline $4,2^{\prime}, 4^{\prime}, 6^{\prime}$-Tetrahydroxychalcone & Flavone & 1.187 & 23.28 & 4.54 & up \\
\hline Narcissoside & Flavonoid & 1.106 & 0.14 & -2.83 & down \\
\hline Orientin & Flavonoid & 1.226 & 23074.07 & 14.49 & up \\
\hline $5,7-$ Dihydroxychromone & Flavonoid & 1.088 & 0.49 & -1.03 & down \\
\hline Herbacetin & Flavonoid & 1.172 & 0.23 & -2.13 & down \\
\hline Isorhamnetin 3-O-glucoside & Flavonoid & 1.227 & 0.00 & -14.36 & down \\
\hline Farrerol & Flavonoid & 1.092 & 0.14 & -2.84 & down \\
\hline Puararin & Flavonoid & 1.225 & 4048.15 & 11.98 & up \\
\hline Diosmin & Flavonoid & 1.225 & 2848.15 & 11.48 & up \\
\hline Tectochrysin & Flavonoid & 1.226 & 44629.63 & 15.45 & up \\
\hline Tectorigenin & Flavonoid & 1.226 & 26111.11 & 14.67 & up \\
\hline Glabridin & Flavonoid & 1.227 & 48370.37 & 15.56 & up \\
\hline Chalcone & Flavonoid & 1.224 & 10122.22 & 13.31 & up \\
\hline
\end{tabular}

Table 2. A list of flavonoid metabolites identified in heartwood and sapwood.

the flavonoids play a crucial role in the formation of wood $\operatorname{color}^{24-26}$. So the flavonoids in HW were selected for further analysis. The results are shown in Table 2, among 313 differential metabolites, we identified a total of 71 flavonoids and isoflavones, including 28 flavones, 3 anthocyanins, 16 flavonols, 11 flavonoids, 8 flavanones and 5 isoflavones. 42 flavonoids were higher and 29 flavonoids lower in HW compared to SW. Among them, the top 5 metabolites that were higher in HW were C-pentosyl-apigenin O-p-coumaroylhexoside, Ayanin, O-methylChrysoeriol 8-C-hexoside, malvidin 3-O-glucoside and chrysin O-hexoside, but the top 5 metabolites significantrly lower in HW compared to SW were $2^{\prime}$-hydroxydaidzein, 8-C-hexosyl chrysoeriol O-hexoside, cyanidin 3-O-malonylhexoside, isorhamnetin 5-O-hexoside and isorhamnetin 3-O-glucoside. These differential flavonoids metabolites show the difference of components between the HW and SW in T. chinensis, but the mechanism for the difference of components between the HW and SW is currently unknown and needs to be further investigated. Moreover, how these differential metabolites affect the wood color of HW also need to be further investigated.

\section{Materials and Methods}

Plant materials. The wood samples were collected from the branch of an approximately 30 -year-old T. chinensis (Pilger) Rehd. in May 2015 from Liangdang County $\left(106^{\circ} 25^{\prime} \mathrm{E}, 33^{\circ} 41^{\prime} \mathrm{N}\right)$ in Shanxi province of China. The HW and SW was separated on the basis of color, the inner wood was HW and the outer SW. Three independent biological replicates were tested for each sample. Samples were dried naturally and ground to a fine powder for usage.

Sample preparation for metabolite profiling. The HW and SW was separated by Tungsten steel knife. The dried HW and SW samples were ground using a mixer mill (MM 400, Retsch). The powder about $100 \mathrm{mg}$ was extracted with $1.0 \mathrm{~mL} 70 \%$ methanol overnight at $4{ }^{\circ} \mathrm{C}$, then the extraction was centrifuged at $10,000 \mathrm{~g}$ for $10 \mathrm{~min}$. The extracts were absorbed and filtrated before LC-MS analysis. The mixed samples of the HW and SW as a quality control were prepared according to the above methods.

Liquid chromatographic mass spectrometry. LC-ESI-MS/MS system (HPLC, Shim-pack UFLC SHIMADZU CBM30A system, MS, Applied Biosystems 6500 Q TRAP) were used to analyze the sample extracts. HPLC analyses used Waters ACQUITY UPLC HSS T3 C18 $(1.8 \mu \mathrm{m}, 2.1 \mathrm{~mm} * 100 \mathrm{~mm})$ column.

solvent system of mobile phase was $0.04 \%$ acetic acid in water and $0.04 \%$ acetic acid in acetonitrile; the gradient program was as follows: $95: 5 \mathrm{~V} / \mathrm{V}$ at $0 \mathrm{~min}, 5: 95 \mathrm{~V} / \mathrm{V}$ at $11.0 \mathrm{~min}, 5: 95 \mathrm{~V} / \mathrm{V}$ at $12.0 \mathrm{~min}, 95: 5 \mathrm{~V} / \mathrm{V}$ at $12.1 \mathrm{~min}$, 95:5 V/V at $15.0 \mathrm{~min}$; the temperature of the column was $40^{\circ} \mathrm{C}$; the injection volume was $2 \mu \mathrm{l}$ and the flow rate was $0.4 \mathrm{ml}$ per minute.

The MS parameter was set as described previously ${ }^{16,18}$. In brief, the temperature of ESI source was $500{ }^{\circ} \mathrm{C}$; the voltage of ion spray was $5500 \mathrm{~V}$; ion source gas I, gas II and curtain gas were set at 55, 60, and 25.0 psi, respectively; the collision gas was set to high. QQQ scans were acquired as MRM experiments with collision gas set to 5 psi. Declustering potential and collision energy for individual MRM transitions was performed with further optimization.

Qualitative and quantitative analysis of metabolites. Based on the MVDB V2.0 Database of Wuhan Maiteville Biotechnology Co., Ltd. (Wuhan, China) and the metabolite information public database ${ }^{27-31}$, the qualitative analysis was performed according to the secondary spectrum information. The isotopic signal is removed during analysis, including $\mathrm{K}^{+}, \mathrm{Na}^{+}, \mathrm{NH}_{4}{ }^{+}$and other fragment ions of large molecular weight substances. The quantitative analysis of metabolites was based on the MRM mode as described previously ${ }^{16,18}$. In the MRM mode, the mass spectrum peak of each different color represented a metabolite. The characteristic ions for each metabolite were filtrated through the triple quadrupole mass spectrometer to obtain the signal strengths. Integration of chromatographic peaks was carried out using MultiQuant. In order to ensure the qualitative and quantitative 
accuracy, the mass spectrum peaks detected in different samples of each metabolite were corrected based on retention time and peak type according to the method described by Fraga et al. ${ }^{32}$.

Statistical analysis. Before analysis, the raw metabolic data were normalized by the method described previously ${ }^{33}$, and the normalized data were $\log _{2}$ transformed before they were used for further analysis. Hierarchical clustering analysis (HCA), principle component analysis (PCA) and partial least squares-discriminant analysis (PLS-DA) have been used to analyze the multivariate and differences of metabolites by soft R (www.r-project. org/) according to the previous study described ${ }^{34,35}$. Based the results of PLS-DA, a fold-change $\geq 2$ or $\leq 0.5$ among the metabolites with a VIP (variable importance in project) value $>1$ was used to identify differential metabolites. The Kyoto Encyclopedia of Genes and Genomes (KEGG) database was used to annotate the differential metabolites and analyze metabolic pathways ${ }^{36}$.

\section{Conclusions}

Wood color is one of the important factors related to wood property. It is critical to understand the chemical compositions that determine the wood color formation. The HW of T. chinensis has high commercial value for its purple red brown color and texture density. To the best of our knowledge, the difference of components between the HW and SW in T. chinensis have not been previously investigated. In this study, the components of the HW and SW in T. chinensis have been analyzed using widely targeted metabolic profiling. A total of 607 metabolites were detected in HW and SW. Among them, 146 metabolites were found significantly higher and 167 metabolites significantly lower in HW as compared to SW. These differential metabolites were mainly involved in metabolic pathways and biosynthesis of secondary metabolites, such as flavonoids, flavone and flavonol, phenylpropanoids and antibiotics. Moreover, the flavonoids in HW associated with wood color were identified. The results provide insight into the metabolites associated with wood color formation and may be useful for understanding the metabolites associated with wood quality.

Received: 20 May 2019; Accepted: 31 October 2019;

Published online: 27 November 2019

\section{References}

1. Chiang, V. From rags to riches. Nat Biotechnol. 20, 557-558 (2002).

2. Ragauskas, A. J. et al. The path forward for biofuels and biomaterials. Science. 311, 484-489 (2006).

3. Hinchee, M. et al. Short-rotation woody crops for bioenergy and biofuels applications. In Vitro Cell Dev Bio Plant. 45, 619-629 (2009).

4. Sarkanen, K. V. Renewable resources for the production of fuels and chemicals. Science. 191, 773-776 (1976).

5. Celedon, J. M. \& Bohlmann, J. An extended model of heartwood secondary metabolism informed by functional genomics. Tree Physiol. 38, 311-319 (2018).

6. Spicer, R. Symplasmic networks in secondary vascular tissues: parenchyma distribution and activity supporting long-distance transport. JExp Bot. 65, 1829-1848 (2014).

7. Miranda, I., Sousa, V., Ferreira, J. \& Pereira, H. Chemical characterization and extractives composition of heartwood and sapwood from Quercus faginea. PLoS One. 12, e0179268 (2017).

8. Kampe, A. \& Magel, E. New insights into heartwood and heartwood formation. (Springer, Berlin, German, 2013).

9. George, B., Suttie, E., Merlin, A. \& Deglise, X. Photodegradation and photostabilisation of wood-The state of the art. Polym Degrad Stab. 88, 268-274 (2005).

10. Sun, Q. F. et al. Preliminary observations of hydrothermal growth of nanomaterials on wood surfaces. Wood Sci Technol. 48, 51-58 (2014).

11. Yao, Q. et al. One-step solvothermal deposition of $\mathrm{ZnO}$ nanorod arrays on a wood surface for robust superamphiphobic performance and superior ultraviolet resistance. Sci Rep. 6, 35505 (2016).

12. Wani, M. C. et al. McPhailPlant antitumor agents. VI. The isolation and structure of taxol, a novel antileukemic and antitumor agent from Taxus brevifolia. J Am Chem Soc. 9, 2325-2327 (1971).

13. Schiff, P. B., Fant, J. \& Horwitz, S. B. Promotion of microtubule assembly in vitro by taxol. Nature. 277, 665-667 (1979).

14. Craag, G. M., Scheparat, S. A., Suffness, M. \& Grever, M. R. The taxol supply crisis. New NCI policies for handling the large-scale production of novel natural product anticancer and anti-HIV agents. J Nat Prod. 56, 1657-1668 (1993).

15. Horwitz, S. B. How to make taxol from scratch. Nature. 367, 593-594 (1994).

16. Wang, D. et al. Identification of Nutritional Components in Black Sesame Determined byWidely Targeted Metabolomics and Traditional Chinese Medicines. Molecules. 23, E1180 (2018).

17. Lu, F. et al. Characterization of eleutheroside B metabolites derived from an extract of Acanthopanax senticosus Harms by highresolution liquid chromatography/quadrupole time-of-flight mass spectrometry and automated data analysis. Biomed. Chromatogr. 26, 1269-1275 (2012).

18. Wu, K. X. et al. A Comparative Metabolomics Analysis Reveals the Tissue-Specific Phenolic Profiling in Two Acanthopanax Species. Molecules. 23, E2078 (2018).

19. Gibbons, H., O'Gorman, A. \& Brennan, L. Metabolomics as a tool in nutritional research. Curr Opin Lipidol. 26, 30-34 (2015).

20. Meijón, M. et al. Exploring natural variation of Pinus pinaster Aiton using metabolomics: is it possible to identify the region of origin of a pine from its metabolites? Mol Ecol. 25, 959-976 (2016).

21. Vogt, T. Phenylpropanoid biosynthesis. Mol Plant. 3, 2-20 (2010).

22. Noel, J. P., Austin, M. B. \& Bomati, E. K. Structure function relationships in plant phenylpropanoid biosynthesis. Curr Opin Plant Biol. 8, 249-253 (2005).

23. Wang, Q. et al. Phenylpropanoids, flavonoids, and terpenoids from Artemisia ordosica Krasch. Magn Reson Chem. 57, 326-330 (2019).

24. Yazaki, Y. Wood Colors and their Coloring Matters: A Review. Nat Prod Commun. 10, 505-12 (2015).

25. Surowiec, I., Nowik, W. \& Trojanowicz, M. Identification of "insoluble" red dyewoods by high performance liquid chromatographyphotodiode array detection (HPLC-PDA) fingerprinting. J Sep Sci. 27, 209-216 (2004).

26. Robertson, A. \& Whalley, W. B. The chemistry of the "insoluble red" woods. VI. Santalin and santarubin. Journal of the Chemical Society. 2794-2801 (1954)

27. Smith, C. A. et al. METLIN: a metabolite mass spectral database. Ther Drug Monit. 27, 747-51 (2005).

28. Horai, H. et al. MassBank: a public repository for sharing mass spectral data for life sciences. J Mass Spectrom. 45, 703-714 (2010). 
29. Afendi, F. M. et al. KNApSAcK family databases: integrated metabolite-plant species databases for multifaceted plant research. Plant Cell Physiol. 53, e1 (2012).

30. Wishart, D. S. et al. HMDB 3.0-The Human Metabolome Database in 2013. Nucleic Acids Res. 41, D801-807 (2013).

31. Moco, S. et al. A liquid chromatography-mass spectrometry-based metabolome database for tomato. Plant Physiol. 141, 1205-1218 (2006).

32. Fraga, C. G., Clowers, B. H., Moore, R. J. \& Zink, E. M. Signature-discovery approach for sample matching of a nerve-agent precursor using liquid chromatography-mass spectrometry, XCMS, and chemometrics. Anal Chem. 82, 4165-4173 (2010).

33. Zhu, G. et al. Rewiring of the Fruit Metabolome in Tomato Breeding. Cell. 172, 249-261 (2018).

34. Thévenot, E. A. et al. Analysis of the Human Adult Urinary Metabolome Variations with Age, Body Mass Index, and Gender by Implementing a Comprehensive Workflow for Univariate and OPLS Statistical Analyses. J Proteome Res. 14, 3322-35 (2015).

35. Wang, S. et al. Spatio-temporal distribution and natural variation of metabolites in citrus fruits. Food Chemistry. 199, 8-17 (2016).

36. Kanehisa, M. et al. KEGG: new perspectives on genomes, pathways, diseases and drugs. Nucleic Acids Res. 45, 353-361 (2017).

\section{Acknowledgements}

The work was supported by the National Key Research \& Development Program of China (Grant Number 2017YFD0600205) and the Natural Science Foundation of China (Grant Numbers 31700584 and 31670676).

\section{Author contributions}

F.S. analyzed the data and performed the experiments; F.S. wrote the manuscript; D.Q. and F.S. designed the experiment; X.L. and W.M. performed sample collection and sample preparation; L.Z., J.G. and I.W. assisted in analyzing the data. All authors have read and approved the version of manuscript.

\section{Competing interests}

The authors declare no competing interests.

\section{Additional information}

Supplementary information is available for this paper at https://doi.org/10.1038/s41598-019-53839-2.

Correspondence and requests for materials should be addressed to D.Q.

Reprints and permissions information is available at www.nature.com/reprints.

Publisher's note Springer Nature remains neutral with regard to jurisdictional claims in published maps and institutional affiliations.

(c) (i) Open Access This article is licensed under a Creative Commons Attribution 4.0 International License, which permits use, sharing, adaptation, distribution and reproduction in any medium or format, as long as you give appropriate credit to the original author(s) and the source, provide a link to the Creative Commons license, and indicate if changes were made. The images or other third party material in this article are included in the article's Creative Commons license, unless indicated otherwise in a credit line to the material. If material is not included in the article's Creative Commons license and your intended use is not permitted by statutory regulation or exceeds the permitted use, you will need to obtain permission directly from the copyright holder. To view a copy of this license, visit http://creativecommons.org/licenses/by/4.0/.

(c) The Author(s) 2019 